\title{
Notes on simplicial rook graphs
}

\author{
Andries E. Brouwer ${ }^{1}$ • Sebastian M. Cioabă $\breve{~}^{2}$ \\ Willem H. Haemers ${ }^{3}$ • Jason R. Vermette ${ }^{4}$
}

Received: 23 August 2014 / Accepted: 25 August 2015 / Published online: 9 September 2015

(c) The Author(s) 2015. This article is published with open access at Springerlink.com

\begin{abstract}
The simplicial rook graph $\mathrm{SR}(m, n)$ is the graph of which the vertices are the sequences of nonnegative integers of length $m$ summing to $n$, where two such sequences are adjacent when they differ in precisely two places. We show that $\operatorname{SR}(m, n)$ has integral eigenvalues, and smallest eigenvalue $s=\max \left(-n,-\left(\begin{array}{c}m \\ 2\end{array}\right)\right)$, and that this graph has a large part of its spectrum in common with the Johnson graph $J(m+n-1, n)$. We determine the automorphism group and several other properties.
\end{abstract}

Keywords Simplicial rook graph · Graph spectra · Integral graph · Johnson graph · Equitable partition

To Chris Godsil, on the occasion of his 65th birthday.

Sebastian M. Cioabă—Research partially supported by the National Security Agency Grant H98230-13-1-0267.

$凶 \quad$ Willem H. Haemers

haemers@uvt.nl

Andries E. Brouwer

aeb@cwi.nl

Sebastian M. Cioabă

cioaba@math.udel.edu

Jason R. Vermette

VermetteJ@mobap.edu

1 Amsterdam, The Netherlands

2 Department of Mathematical Sciences, University of Delaware, Newark, DE 19716-2553, USA

3 Department of Econometrics and Operations Research, Tilburg University,

Tilburg, The Netherlands

4 Natural Sciences Division, Missouri Baptist University, Saint Louis, MO 63141-8698, USA 


\section{Introduction}

Let $\mathbb{N}$ be the set of nonnegative integers, and let $m, n \in \mathbb{N}$. The simplicial rook graph $\operatorname{SR}(m, n)$ is the graph $\Gamma$ obtained by taking as vertices the vectors in $\mathbb{N}^{m}$ with coordinate sum $n$, and letting two vertices be adjacent when they differ in precisely two coordinate positions. Then $\Gamma$ has $v=\left(\begin{array}{c}n+m-1 \\ n\end{array}\right)$ vertices, and is regular of valency $k=n(m-1)$.

Small cases: For $n>0, m=0$, the graph is $K_{0}$. For $n=0$ or $m=1$, the graph is $K_{1}$. For $n=1$, the graph is $K_{m}$. For $m=2$, the graph is $K_{n+1}$. For $n=2$, the graph is the triangular graph $T(m+1)$.

The graph $\operatorname{SR}(m, n)$ was studied in detail by Martin and Wagner [9]. Here we settle their main conjecture and show that this graph has integral spectrum. Some results:

Theorem 2.1 The graph $\operatorname{SR}(m, n)$ has integral spectrum.

Proposition 3.1 If $\mathrm{SR}(m, n)$ has at least one vertex, it has smallest eigenvalue $\max \left(-n,-\left(\begin{array}{c}m \\ 2\end{array}\right)\right)$.

The Johnson graph $J(m, n)$ [with $\left(\begin{array}{l}m \\ n\end{array}\right)$ vertices, valency $n(m-n)$ and eigenvalues $(n-i)(m-n-i)-i$ with multiplicity $\left(\begin{array}{c}m \\ i\end{array}\right)-\left(\begin{array}{c}m \\ i-1\end{array}\right)(0 \leq i \leq n)$, cf. [2]] is an induced subgraph of $\Gamma$ (on the set of vertices in $\{0,1\}^{m}$ ).

The graphs $\operatorname{SR}(m, n)$ and $J(m+n-1, n)$ both have $\left(\begin{array}{c}m+n-1 \\ n\end{array}\right)$ vertices and valency $n(m-1)$. These graphs resemble each other and have a large part of their spectrum in common.

Proposition 5.1 The graphs $\operatorname{SR}(m, n)$ and $J(m+n-1, n)$ have equitable partitions with the same quotient matrix $E$, where $E$ has eigenvalues $(n-i)(m-i)-n$ with multiplicity $\left(\begin{array}{c}m \\ i\end{array}\right)$ for $0 \leq i<n$, and multiplicity $\left(\begin{array}{c}m \\ n\end{array}\right)-1$ for $i=n$. In particular, the spectrum of $E$ is part of the spectrum of $\operatorname{SR}(m, n)$ and $J(m+n-1, n)$.

Clearly, $\operatorname{Sym}(m)$ acts as a group of automorphisms, permuting the coordinate positions. Except for small cases, this is the full group.

Proposition 6.1 Let $m, n>2$. If $n>3$, then $\operatorname{Aut}(\Gamma) \simeq \operatorname{Sym}(m)$. If $n=3$, then $\operatorname{Aut}(\Gamma) \simeq \operatorname{Sym}(m) \cdot 2$.

We determine some miscellaneous properties.

Proposition 7.1 Let $m>0$. Then the diameter of $\operatorname{SR}(m, n)$ is $\min (m-1, n)$.

Proposition 8.2 Let $m>1$ and $n>0$. Then the size of the largest clique in $\mathrm{SR}(m, n)$ is $\max (m, n+1)$.

We also give the independence number in case $n=3$, and the complete spectrum for a few small $n$. In general, the graphs $\operatorname{SR}(m, n)$ are not determined by their spectrum.

Proposition 10.1 The graph $\mathrm{SR}(m, n)$ is not determined by its spectrum when $(a)$ $m=4$ and $n \geq 3$, or $(b) n=3$ and $m \geq 4$.

Finally, we study the structure of the eigenspace for the eigenvalue $-n$. 


\section{Integrality of the eigenvalues}

We start with the main result, proved by a somewhat tricky induction.

Theorem 2.1 All eigenvalues of $\operatorname{SR}(m, n)$ are integers.

Proof Let $\Gamma$ be the graph SR $(m, n)$, and let $X$ be its vertex set. The adjacency matrix $A$ of $\Gamma$ acts as a linear operator on $\mathbb{R}^{X}$ (sending each vertex to the sum of its neighbors). By induction, we construct a series of subspaces $0=U_{0} \subseteq U_{1} \subseteq \ldots \subseteq U_{t}=\mathbb{R}^{X}$ and find integers $c_{i}$, such that $\left(A-c_{i} I\right) U_{i} \subseteq U_{i-1}(1 \leq i \leq t)$. Then $p(A):=\prod_{i}\left(A-c_{i} I\right)$ vanishes identically, and all eigenvalues of $A$ are among the integers $c_{i}$.

For $j \neq k$, let $A_{j k}$ be the matrix that describes adjacency between vertices that differ only in the $j$ - and $k$-coordinates. Then $A=\sum A_{j k}$. If $\left(A_{j k}-c_{j k} I\right) u \in U$ for all $j, k$ then $(A-c I) u \in U$ for $c=\sum c_{j k}$.

A basis for $\mathbb{R}^{X}$ is given by the vectors $e_{x}(x \in X)$ that have $y$-coordinate 0 for $y \neq x$, and $x$-coordinate 1 . For $S \subseteq X$, let $e_{S}:=\sum_{x \in S} e_{x}$, so that $e_{X}$ is the all-1 vector. Since $(A-c I) e_{X}=0$ for $c=(m-1) n$, we can put $U_{1}=\left\langle e_{X}\right\rangle$.

For partitions $\Pi$ of the set of coordinate positions $\{1, \ldots, m\}$ and integral vectors $z$ indexed by $\Pi$ that sum to $n$, let $S_{\Pi, z}$ be the set of all $u \in X$ with $\sum_{i \in \pi} u_{i}=z_{\pi}$ for all $\pi \in \Pi$. If $\Pi$ is a partition into singletons, then $\left|S_{\Pi, z}\right|=1$.

For a vector $y$ indexed by a partition $\Pi$, let $\tilde{y}$ be the sequence of pairs $\left(y_{\pi},|\pi|\right)$ $(\pi \in \Pi)$ sorted lexicographically: with the $y_{\pi}$ in nondecreasing order, and for given $y_{\pi}$ with the $|\pi|$ in nondecreasing order.

Order pairs $(\Pi, z)$ by $(\Sigma, y)<(\Pi, z)$ when $|\Sigma|<|\Pi|$, or when $|\Sigma|=|\Pi|$ and $\tilde{y} \neq \tilde{z}$ and in the first place $j$ where $\tilde{y}$ and $\tilde{z}$ differ, the pair $\tilde{y}_{j}$ is lexicographically smaller than the pair $\tilde{z}_{j}$.

We use induction to show for $S=S_{\Pi, z}$ and suitable $c$ that the image $(A-c I) e_{S}$ lies in the subspace $U$ spanned by $e_{T}$ for $T=S_{\Sigma, y}$, where $(\Sigma, y)<(\Pi, z)$.

Note that the sets $S=S_{\Pi, z}$ induce regular subgraphs of $\Gamma$. Indeed, the induced subgraph is a copy of the Cartesian product $\prod_{\pi} \operatorname{SR}\left(|\pi|, z_{\pi}\right)$. The image $(A-c I) e_{S}$ can be viewed as a multiset where the $x \in X$ occur with certain multiplicities. The fact that $S$ induces a regular subgraph means that we can adjust $c$ to give all $x \in S$ any desired given multiplicity, while the multiplicity of $x \notin S$ does not depend on $c$.

If $j, k$ belong to the same part of $\Pi$, then $A_{j k} e_{S}$ only contains points of $S$ and can be ignored. So, let $j \in \pi, k \in \rho$, where $\pi, \rho \in \Pi, \pi \neq \rho$, and consider $A_{j k} e_{S}$. Abbreviate $\pi \cup\{k\}$ with $\pi+k$ and $\pi \backslash\{j\}$ with $\pi-j$.

The image $\left(A_{j k}-c I\right) e_{S}$ equals $S_{1}-S_{2}$, where $S_{1}$ is the sum of all $e_{T}$, with $T=S_{\Sigma, y}$ and $\Sigma=(\Pi \backslash\{\pi, \rho\}) \cup\{\pi-j, \rho+j\}$ (omitting $\pi-j$ if it is empty) and $y$ agrees with $z$ except that $y_{\pi-j} \leq z_{\pi}$ and $y_{\rho+j} \geq z_{\rho}$ (of course $y_{\pi-j}+y_{\rho+j}=z_{\pi}+z_{\rho}$ ), and $S_{2}$ is the sum of all $e_{T}$, with $T=S_{\Sigma, y}$ and $\Sigma=(\Pi \backslash\{\pi, \rho\}) \cup\{\pi+k, \rho-k\}$ and $y$ agrees with $z$ except that $y_{\pi+k}<z_{\pi}$ and $y_{\rho-k}>z_{\rho}$.

[Let $u$ be a $(j, k)$-neighbor of $s \in S$. Since $\sum_{i \in \pi} s_{i}=z_{\pi}$, it follows that $\sum_{i \in \pi-j} u_{i}=\sum_{i \in \pi-j} s_{i} \leq z_{\pi}$, so that $u$ is counted in $S_{1}$. Conversely, if $u$ is counted in $S_{1}$, then we find a $(j, k)$-neighbor $s \in S$ by moving $u_{j}-s_{j}$ from position $j$ to position $k$ (if $u_{j}>s_{j}$ ) or moving $s_{j}-u_{j}$ from position $k$ to position $j$ (if $s_{j}>u_{j}$ ). The latter is impossible if $u_{k}<s_{j}-u_{j}$, i.e. $\sum_{i \in \pi+k} u_{i}<z_{\pi}$, and these cases are subtracted in $S_{2}$.] 
We are done by induction. Indeed, for the pair $\{j, k\}$ we can choose which of the two is called $j$, and we pick notation such that $\left(z_{\pi},|\pi|\right) \leq\left(z_{\rho},|\rho|\right)$ in lexicographic order. Now in $S_{1}$ and $S_{2}$ only $(\Sigma, y)$ occur with $(\Sigma, y)<(\Pi, z)$.

\section{The smallest eigenvalue}

We find the smallest eigenvalue of $\Gamma$ by observing that $\Gamma$ is a halved graph of a bipartite graph $\Delta$.

Consider the bipartite graph $\Delta$ of which the vertices are the vectors in $\mathbb{N}^{m}$ with coordinate sum at most $n$, where two vertices are adjacent when one has coordinate sum $n$, the other coordinate sum less than $n$, and both differ in precisely one coordinate. Let $V$ be the set of vectors in $\mathbb{N}^{m}$ with coordinate sum $n$. Two vectors $u, v$ in $V$ are adjacent in $\Gamma$ precisely when they have distance 2 in $\Delta$. If the adjacency matrix of $\Delta$ is $\left(\begin{array}{ll}0 & N \\ N^{\top} & 0\end{array}\right)$, with top and left indexed by $V$, then for the adjacency matrix $A$ of $\Gamma$ we find $A+n I=N N^{\top}$, so that $A+n I$ is positive semidefinite, and the smallest eigenvalue of $A$ is not smaller than $-n$.

Together with the results of [9], this proves that the smallest eigenvalue of $A$ equals $\max \left(-n,-\left(\begin{array}{c}m \\ 2\end{array}\right)\right)$.

Proposition 3.1 If $\mathrm{SR}(m, n)$ has at least one vertex, it has smallest eigenvalue $\max \left(-n,-\left(\begin{array}{c}m \\ 2\end{array}\right)\right)$.

Proof Let $s$ be the smallest eigenvalue of $A$. We just saw that $s \geq-n$. Elkies [6] observed that $s \geq-\left(\begin{array}{c}m \\ 2\end{array}\right)$, since $A$ is the sum of $\left(\begin{array}{c}m \\ 2\end{array}\right)$ matrices $A_{j k}$ that describe adjacency where only coordinates $j, k$ are changed. Each $A_{j k}$ is the adjacency matrix of a graph that is a union of cliques and hence has smallest eigenvalue not smaller than -1 . Then $A=\sum A_{j k}$ has smallest eigenvalue not smaller than $-\left(\begin{array}{c}m \\ 2\end{array}\right)$.

It is shown in [9] that the eigenvalue $-\left(\begin{array}{c}m \\ 2\end{array}\right)$ has multiplicity at least $\left(\begin{array}{c}n-\left(\begin{array}{c}m-1 \\ 2 \\ m-1\end{array}\right) \\ m-1\end{array}\right)$ and hence occurs with nonzero multiplicity if $n \geq\left(\begin{array}{c}m \\ 2\end{array}\right)$. It is also shown in [9] that the multiplicity of the eigenvalue $-n$ is at least the number of permutations in $\operatorname{Sym}(m)$ with precisely $n$ inversions, that is the number of words $w$ of length $n$ in this Coxeter group, and this is nonzero precisely when $n \leq\left(\begin{array}{c}m \\ 2\end{array}\right)$.

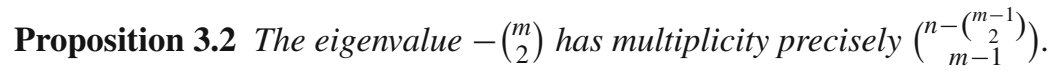

Proof For each vertex $u$, and $1 \leq j<k \leq m$, let $C_{j k}(u)$ be the $(j, k)$-clique on $u$, that is the set of all vertices $v$ with $v_{i}=u_{i}$ for $i \neq j, k$. An eigenvector $a=\left(a_{u}\right)$ for the eigenvalue $-\left(\begin{array}{c}m \\ 2\end{array}\right)$ must be a common eigenvector of all $A_{j k}$ for the eigenvalue -1 . That means that $\sum_{v \in C} a_{v}=0$ for each set $C=C_{j k}(u)$.

Order the vertices by $u>v$ when $u_{d}>v_{d}$ when $d=d_{u v}$ is the largest index where $u, v$ differ. Suppose $u_{i}=s$ for some index $i$ and $s \leq m-i-1$. We can express $a_{u}$ in terms of $a_{v}$ for smaller $v$ with $d_{u v} \geq m-s$ via $\sum_{v \in C} a_{v}=0$, where $C=C_{i, m-s}(u)$. Indeed, this equation will express $a_{u}$ in terms of $a_{v}$ where $u_{i}+u_{m-s}=v_{i}+v_{m-s}$ and $v_{j}=u_{j}$ for $j \neq i, m-s$. If $v_{i}>s$, this is OK since $v_{m-s}<u_{m-s}$. If $t=v_{i}<s$, 
then by induction $a_{v}$ in its turn can be expressed in terms of $a_{w}$ where $w$ is smaller and $d_{v w} \geq m-t>m-s$, so that $w$ is smaller than $u$, and $d_{u w}>m-s$.

In this way, we expressed $a_{u}$ when $u_{i} \leq m-i-1$ for some $i$. The free $a_{u}$ have $u_{i} \geq m-i$ for all $i$, and the vector $u^{\prime}$ with $u_{i}^{\prime}=u_{i}-(m-i)$ is nonnegative and sums to $n-\left(\begin{array}{c}m \\ 2\end{array}\right)$. There are $\left(\begin{array}{c}n-\left(\begin{array}{c}m \\ 2\end{array}\right)+m-1 \\ m-1\end{array}\right)=\left(\begin{array}{c}n-\left(\begin{array}{c}m-1 \\ 2 \\ m-1\end{array}\right) \\ \text { - }\end{array}\right)$ such vectors, so this is an upper bound for the multiplicity. But by [9] this is also a lower bound.

Thanks to a suggestion by Aart Blokhuis, we can also settle the multiplicity of the eigenvalue $-n$.

Proposition 3.3 The multiplicity of the eigenvalue $-n$ equals the number of elements of $\operatorname{Sym}(m)$ with $n$ inversions, that is, the coefficient of $t^{n}$ in the product $\prod_{i=2}^{m}(1+t+$ $\left.\cdots+t^{i-1}\right)$.

Proof As already noted, it is shown in [9] that the multiplicity of the eigenvalue $-n$ is at least the number of permutations in $\operatorname{Sym}(m)$ with precisely $n$ inversions. The formula $\sum_{w} t^{l(w)}=\prod_{i=2}^{m} \frac{t^{i}-1}{t-1}$, where $w$ runs over $\operatorname{Sym}(m)$ and $l(w)$ is the number of inversions of $w$, is standard, cf. [8], p. 73.

Since $A+n I=N N^{\top}$, the multiplicity of the eigenvalue $-n$ is the nullity of $N$, and we need an upper bound for that.

We first define a matrix $P$ and observe that $N$ and $P$ have the same column space and hence the same rank. For $u, v \in \mathbb{N}^{m}$, write $u \preceq v$ when $u_{i} \leq v_{i}$ for all $i$. Let $P$ be the 0-1 matrix with the same row and column indices (elements of $\mathbb{N}^{m}$ with sum $m$ and sum smaller than $m$, respectively) where $P_{x y}=1$ when $y \preceq x$. Recall that $N$ is the $0-1$ matrix with $N_{x y}=1$ when $x$ and $y$ differ in precisely one coordinate position. Let $M(y)$ denote column $y$ of the matrix $M$.

For $d=n-\sum y_{i}$, we find that

$$
N(y)=\sum_{i=0}^{d-1}(-1)^{i}(d-i) \sum_{z \in W_{i}} P(y+z),
$$

where $W_{i}$ is the set of vectors in $\{0,1\}^{m}$ with sum $i$. Indeed, suppose that $x$ and $y$ differ in $j$ positions. Then $j \leq d$, and $N_{x y}=\delta_{1 j}$, while the $x$-entry of the right-hand side is $\sum_{i=0}^{d}(-1)^{i}(d-i)\left(\begin{array}{l}j \\ i\end{array}\right)=j \sum_{i=1}^{j}(-1)^{i-1}\left(\begin{array}{l}j-1 \\ i-1\end{array}\right)=j(1-1)^{j-1}=\delta_{1 j}$. We see that $N(y)$ and $d P(y)$ differ by a linear combination of columns $P\left(y^{\prime}\right)$ where $\sum y_{i}^{\prime}>\sum y_{i}$, and hence that $N$ and $P$ have the same column space.

Aart Blokhuis remarked that the coefficient of $t^{n}$ in the product $\prod_{i=2}^{m}(1+t+\cdots+$ $t^{i-1}$ ) is precisely the number of vertices $u$ satisfying $u_{i}<i$ for $1 \leq i \leq m$. Thus, it suffices to show that the rows of $N$ (or $P$ ) indexed by the remaining vertices are linearly independent.

Consider a linear dependence between the rows of $P$ indexed by the remaining vertices, and let $P^{\prime}$ be the submatrix of $P$ containing the rows that occur in this dependence. Order vertices in reverse lexicographic order, so that $u$ is earlier than $u^{\prime}$ when $u_{h}<u_{h}^{\prime}$ and $u_{i}=u_{i}^{\prime}$ for $i>h$. Let $x$ be the last row index of $P^{\prime}$ (in this order). Let $h$ be an index where the inequality $x_{i}<i$ is violated, so that $x_{h} \geq h$. Let $e_{i}$ be the 
element of $\mathbb{N}^{m}$ that has all coordinates 0 except for the $i$-coordinate, which is 1 . Let $z=x-h e_{h}$. Let $H=\{1, \ldots, h-1\}$. For $S \subseteq H$, let $\chi(S)$ be the element of $\mathbb{N}^{m}$ that has $i$-coordinate 1 if $i \in S$, and 0 otherwise.

Consider the linear combination $p=\sum_{S}(-1)^{|S|} P^{\prime}(z+\chi(S))$ of the columns of $P^{\prime}$. We shall see that $p$ has $x$-entry 1 and all other entries equal to 0 . But that contradicts the existence of a linear dependence.

If $u$ is a row index of $P^{\prime}$, and not $z \preceq u$, then $p_{u}=0$. If $z \preceq u$, and $z_{i}<u_{i}$ for some $i<h$, then the alternating sum vanishes, and $p_{u}=0$. So, if $p_{u} \neq 0$, then $u$ agrees with $x$ in coordinates $i, 1 \leq i \leq h-1$. For row $x$ only $S=\emptyset$ contributes, and $p_{x}=1$. Finally, if $u \neq x$, then $u_{i} \geq x_{i}$ for $i \neq h$ and $\sum x_{i}=\sum u_{i}=n$ imply that $u_{h}<x_{h}$ and $u_{i}>x_{i}$ for some $i>h$, which is impossible, since $x$ is the reverse lexicographically latest row index of $P^{\prime}$.

These three propositions settle conjectures from [9].

\section{An equitable partition}

A partition $\left\{X_{1}, \ldots, X_{t}\right\}$ of the vertex set $X$ of a graph $\Gamma$ is called equitable when for all $i, j$ the number $e_{i j}$ of vertices in $X_{j}$ adjacent to a given vertex $x \in X_{i}$ does not depend on the choice of $x \in X_{i}$. In this case, the matrix $E=\left(e_{i j}\right)$ is called the quotient matrix of the partition. All eigenvalues of $E$ are also eigenvalues of $\Gamma$, realized by eigenvectors that are constant on the sets $X_{i}$. There is a basis of $\mathbb{R}^{X}$ consisting of eigenvectors that either are constant on all $X_{i}$ or sum to zero on all $X_{i}$. The partition of $X$ into orbits of an automorphism group $G$ of $\Gamma$ is always equitable.

In this section, we indicate an equitable partition of $\operatorname{SR}(m, n)$, and in the next section a much finer one.

Let $\Gamma$ be the graph $\operatorname{SR}(m, n)$ where $n>0$, and let $V_{i}$ be the set of vertices with precisely $i$ nonzero coordinates, so that $\left|V_{i}\right|=\left(\begin{array}{c}m \\ i\end{array}\right)\left(\begin{array}{c}n-1 \\ i-1\end{array}\right)$.

Proposition 4.1 The partition $\left\{V_{1}, \ldots, V_{\min (m, n)}\right\}$ is equitable. Each $x \in V_{i}$ has $i(i-1)$ neighbors in $V_{i-1},(n-i)(m-i)$ neighbors in $V_{i+1}$, and all other neighbors in $V_{i}$. The quotient matrix $E$ is tridiagonal and has eigenvalues $(m-i)(n-i)-n$ for $0 \leq i \leq \min (m, n)-1$.

Proof The $e_{i j}$ are easily checked. It remains to find the eigenvalues. Let $u$ be an eigenvector for the Johnson graph $J(m+n-1, n)$ for the eigenvalue $\theta=(m-$ $i)(n-i)-n$. Then $c_{i} u_{i-1}+\left(k-c_{i}-b_{i}\right) u_{i}+b_{i} u_{i+1}=\theta u_{i}$, where $c_{i}=i^{2}$ and $b_{i}=(m-1-i)(n-i)$ and $k=(m-1) n$. Define $v_{i}=i u_{i-1}+(m-i) u_{i}$ $(1 \leq i \leq n)$. Then $c_{i}^{\prime} v_{i-1}+\left(k-c_{i}^{\prime}-b_{i}^{\prime}\right) v_{i}+b_{i}^{\prime} v_{i+1}=\theta v_{i}$, where $c_{i}^{\prime}=i(i-1)$ and $b_{i}^{\prime}=(n-i)(m-i)$. It follows that $E v=\theta v$. We have $v \neq 0$ for $\theta \neq-n$.

Remark The Johnson graph $J(m, n)$ has eigenvalues $(n-i)(m+1-n-i)-n$ with multiplicity $\left(\begin{array}{c}m \\ i\end{array}\right)-\left(\begin{array}{c}m \\ i-1\end{array}\right)(0 \leq i \leq \min (n, m-n))$. It is not wrong to say that it has these eigenvalues and multiplicities for $0 \leq i \leq n$ since by convention the multiplicities of an eigenvalue are added, and eigenvalues with multiplicity 0 are no eigenvalues. For example, $J(5,4)$ has spectrum $4^{1}(-1)^{4}$, which is the same as $4^{1}(-1)^{4}(-4)^{5}(-5)^{0}(-4)^{-5}$, where multiplicities are written as exponents. The 
Johnson graph $J(m+n-1, n)$ has eigenvalues $(m-i)(n-i)-n$ with multiplicity $\left(\begin{array}{c}m+n-1 \\ i\end{array}\right)-\left(\begin{array}{c}m+n-1 \\ i-1\end{array}\right)(0 \leq i \leq \min (n, m-1))$.

\section{The common part of the spectra of $\operatorname{SR}(m, n)$ and $J(m+n-1, n)$}

Both $\operatorname{SR}(m, n)$ and $J(m+n-1, n)$ have $\left(\begin{array}{c}m+n-1 \\ n\end{array}\right)$ vertices. Both have valency $n(m-1)$. These graphs resemble each other and have a large part of their spectrum in common. Let $m, n>0$.

Proposition 5.1 The graphs $\mathrm{SR}(m, n)$ and $J(m+n-1, n)$ have equitable partitions with the same quotient matrix $E$, where $E$ has eigenvalues $(n-i)(m-i)-n$ with multiplicity $\left(\begin{array}{c}m \\ i\end{array}\right)$ for $0 \leq i \leq n-1$, and multiplicity $\left(\begin{array}{c}m \\ n\end{array}\right)-1$ for $i=n$. In particular, the spectrum of $E$ is a common part of the spectrum of $\operatorname{SR}(m, n)$ and that of $J(m+n-1, n)$.

That is, $E$ has eigenvalue $(n-i)(m-i)-n$ with multiplicity $\left(\begin{array}{c}m \\ i\end{array}\right)$ for $0 \leq i \leq$ $\min (m, n)-1$, and if $n<m$ also eigenvalue $-n$ with multiplicity $\left(\begin{array}{c}m \\ n\end{array}\right)-1$.

Proof Partition the vertex set of $\operatorname{SR}(m, n)$ into $\sum_{i=1}^{n}\left(\begin{array}{c}m \\ i\end{array}\right)$ parts, where each part consists of the vertices with fixed support $S$ (of weight $i$ ). Partition the vertex set of $J(m+n-1, n)$ into $\sum_{i=1}^{n}\left(\begin{array}{c}m \\ i\end{array}\right)$ parts, where each part consists of the vertices with fixed support $S$ (of weight $i$ ) in the first $m$ coordinates. The size of part $S$, where $|S|=i$, is $\left(\begin{array}{l}n-1 \\ n-i\end{array}\right)$ in both cases.

It is straightforward to determine the numbers $e_{S T}$ for both graphs. For $\operatorname{SR}(m, n)$, note that if we restrict attention to vertices with support $S$, where $|S|=i$, then our graph becomes a copy of $\operatorname{SR}(i, n-i)$, as one sees by subtracting 1 from all entries. The result is

$$
e_{S T}= \begin{cases}(i-1)(n-i) & \text { if }|S|=i, S=T \\ i-1 & \text { if }|S|=i,|T|=i-1, S \supset T \\ n-i & \text { if }|S|=i,|T|=i+1, S \subset T \\ 1 & \text { if }|S|=|T|=i \text { and } S, T \text { differ in two places } \\ 0 & \text { otherwise, }\end{cases}
$$

in both cases. It follows that our partitions are equitable with the same quotient matrix $E$. We may conclude that $J(m+n-1, n)$ and $\operatorname{SR}(m, n)$ have the $\sum_{i=1}^{n}\left(\begin{array}{c}m \\ i\end{array}\right)$ eigenvalues of the matrix $E$ in common.

Claim: These eigenvalues are $(n-i)(m-i)-n$ with multiplicity $\left(\begin{array}{c}m \\ i\end{array}\right)$ for $0 \leq i<n$, and multiplicity $\left(\begin{array}{l}m \\ n\end{array}\right)-1$ for $i=n$. These are the eigenvalues of $J(m+n-1, n)$, so we need only confirm the multiplicities.

Let $W_{i j}$ be the (symmetrized) inclusion matrix of $i$-subsets against $j$-subsets in a $v$-set. Then $W_{i j}=W_{j i}^{\top}$. For $h \leq i \leq j$, we have $W_{h i} W_{i j}=\left(\begin{array}{c}j-h \\ i-h\end{array}\right) W_{h j}$. Also $W_{i i}=I$ and $W_{i, j+1} W_{j+1, j}=W_{i, i-1} W_{i-1, j}+(v-i-j) W_{i, j}$ for $j \geq i$. (Note that $W_{i,-1}$ has no columns, and $W_{-1, j}$ has no rows, so that $\left.W_{i,-1} W_{-1, j}=0\right)$.

For $0 \leq i \leq n$, the $i$ th eigenspace of $J(v, n)$ is spanned by vectors $W_{n i} w$ where $w$ is indexed by the $i$-subsets of the $v$-set and $W_{i-1, i} w=0$ if $i>0$. (Indeed, the adjacency matrix of $J(v, n)$ is $A=W_{n, n-1} W_{n-1, n}-n I$, and $W_{n, n-1} W_{n-1, n} W_{n i} w=$ 
$W_{n, n-1}\left(W_{n-1, i-1} W_{i-1, i}+(v-n-i+1) W_{n-1, i}\right) w=(v-n-i+1)(n-i) W_{n i} w$, so that $J(v, n)$ has eigenvalue $(v-n-i+1)(n-i)-n$ with multiplicity $\left.\left(\begin{array}{c}v \\ i\end{array}\right)-\left(\begin{array}{c}v \\ i-1\end{array}\right)\right)$.

Let $v=m+n-1$, and consider the vectors $W_{n i} w$ that are invariant under $\operatorname{Sym}(n-1)$ acting on the final $n-1$ coordinates. They form a space of dimension $\sum_{j=0}^{i}\left(\begin{array}{c}m \\ j\end{array}\right)$ if $i<n$, and $\sum_{j=1}^{n}\left(\begin{array}{c}m \\ j\end{array}\right)$ if $i=n$. The subspace of such vectors satisfying $W_{i-1, i} w=0$ has codimension $\sum_{j=0}^{i-1}\left(\begin{array}{c}m \\ j\end{array}\right)$ : Each $(i-1)$-set $T$ imposes a restriction $\left(W_{i-1, i} w\right)_{T}=0$, and such restrictions are equivalent when the $T$ 's are in the $\operatorname{same} \operatorname{Sym}(n-1)$-orbit. It follows that the corresponding eigenspace of $E$ has dimension $\left(\begin{array}{c}m \\ i\end{array}\right)$ when $i<n$. For $i=n$, this same computation becomes $\left(\sum_{j=1}^{n}\left(\begin{array}{c}m \\ j\end{array}\right)\right)-\left(\sum_{j=0}^{n-1}\left(\begin{array}{c}m \\ j\end{array}\right)\right)=\left(\begin{array}{c}m \\ n\end{array}\right)-1$.

\section{Automorphism group}

Clearly, $G=\operatorname{Sym}(m)$ acts as a group of automorphisms on $\Gamma$, permuting the coordinate positions. This action is faithful, unless $n=0, m>1$ when the full group has order 1 . When $m=2$, the graph is $K_{n+1}$ and the full group is $\operatorname{Sym}(n+1)$. When $n=2$, the graph is $T(m+1) \simeq J(m+1,2)$ with full group $\operatorname{Sym}(m+1)$ for $m \neq 1,3$ and $2^{3} \cdot \operatorname{Sym}(3)$ for $m=3$.

Proposition 6.1 Let $m, n>2$. If $n=3$, then $\operatorname{Aut}(\Gamma) \simeq \operatorname{Sym}(m) .2$, where the additional factor 2 interchanges the digits 1 and 2 in each vector with a coordinate 2. If $n>3$, then $\operatorname{Aut}(\Gamma) \simeq \operatorname{Sym}(m)$.

Proof Classify the vertices $x$ according to the number $\lambda_{x y}$ of common neighbors of $x$ and $y$, for all neighbors $y$ of $x$.

We always have $\lambda_{x y} \leq m+n-3$. (Indeed, look at the common neighbors of $x=$ $(a, b, c, \ldots)$ and $y=(a+d, b-d, c, \ldots)$. We find $\left(a^{\prime}, b^{\prime}, c, \ldots\right)$ with $a^{\prime}+b^{\prime}=a+b$ $(a+b-1$ choices $)$, and $(a, b-d, c+d, \ldots)(m-2$ choices $)$, and $(a+d, b, c-d, \ldots)$ where the number of choices is the number of $c$ not less than $d$. If $a+b=n$, there are no such $c$. Otherwise, this number is maximal when $d=1$ and all nonzero $c$ equal 1 and then equals $n-a-b$. So $\lambda_{x y} \leq a+b-1+m-2+n-a-b=m+n-3$.)

Note for later use the structure of the graph $\Lambda(x, y)$ induced by the common neighbors of $x$ and $y$. It is $K_{a+b-1}+K_{m-2}+K_{g}$, where $g$ is the number of $c$ (common coordinates of $x$ and $y$ ) not less than $d$.

If $\lambda_{x y}=m+n-3$ for all $n(m-1)$ neighbors $y$ of $x$ (and $m>2$ ), then $x$ has either a unique nonzero coordinate $n$ or only coordinates 0,1 . Thus, we can recognize this set of $m+\left(\begin{array}{c}m \\ n\end{array}\right)$ vertices. The induced subgraph (for $n>2$ ) is isomorphic to $K_{m}+J(m, n)$.

We see that $\Gamma$ determines $m+n-3$ and also the pair $\left\{m,\left(\begin{array}{c}m \\ n\end{array}\right)\right\}$. Now $m$ is the smallest element of the pair distinct from 0,1 , so we find $m$ and $n$.

Suppose first that $n \neq m-1$. Then we recognized the set $S$ of vectors with a unique nonzero coordinate. At distance $i$ from $S$ lie the vectors with precisely $i+1$ nonzero coordinates, and the positions of the nonzero coordinates of a vector $u$ are determined by the set of nearest vertices in $S$. We show by induction on $m$ that all vertex labels are determined. If a vertex $(a, b, \ldots)$ has at least two nonzero coordinates, and $m>3$, then its neighbor $(0, a+b, \ldots)$ lies in the $\operatorname{SR}(m-1, n)$ on the vertices with first coordinate zero, and by induction $a+b$ is determined. If it has at least three 
nonzero coordinates: $(a, b, c, \ldots)$, then each of $a+b, a+c, b+c$ is determined and hence also $a, b, c$. If it has precisely two nonzero coordinates: $(a, n-a, 0, \ldots)$, then it has neighbors $(a-i, n-a, i, 0, \ldots)(1 \leq i \leq a-1)$ and $(a, n-a-j, j, 0, \ldots)$ $(1 \leq j \leq n-a-1)$ of which all coordinates are known, and $a$ and $n-a$ follow unless $\{a, n-a\}=\{1,2\}$. This settles all claims when $m>3, n \neq m-1$.

If $m=3, n \geq 3$, we recall that the common neighbors of vertices $x$ and $y$ induce $K_{a+b-1}+K_{m-2}+K_{g}$, where $m-2=1$ and $g \leq 1$, so that $a+b$ can be recognized directly when $a+b \geq 3$. But we also know the zero pattern, so can also recognize $a, b$ when $a+b \leq 2$. This determines all for $m=3$.

Finally, if $m=n+1 \geq 4$, we have to distinguish the copy of $K_{m}$ on the vectors of shape $n^{1} 0^{n}$ from that on the vectors of shape $1^{n} 0$. Both sets have $m\left(\begin{array}{c}m-1 \\ 2\end{array}\right)$ neighbors, but if $x=(n, 0, \ldots)$ and $y=(n-a, a, \ldots)$, then $\Lambda(x, y) \simeq 2 K_{n-1}$, while $\Lambda(x, y) \simeq$ $K_{n-1}+K_{n-2}+K_{1}$ if $x=(1, \ldots, 1,1,0)$ and $y=(2,1, \ldots, 1,0,0)$. This settles all cases.

\section{Diameter}

Proposition 7.1 Let $m>0$. Then the diameter of $\operatorname{SR}(m, n)$ is $\min (m-1, n)$.

Proof The diameter is at most $m-1$, since one can walk from one vertex to another and decrease the number of different coordinates by at least one at each step. The diameter is also at most $n$, since one can walk from one vertex to another and decrease the sum of the absolute values of the coordinate differences by at least two at each step. If $m>n$, then $(0, \ldots, 0, n)$ and $(1, \ldots, 1,0, \ldots, 0)$ show that the diameter is at least $n$. If $m \leq n$, then $(0, \ldots, 0, n)$ and $(1, \ldots, 1, n-m+1)$ show that the diameter is at least $m-1$.

\section{Maximal cliques and local graphs}

We classify the cliques (complete subgraphs) and find the maximal ones. We also examine the structure of the local graphs of $\Gamma$.

Lemma 8.1 Cliques $C$ in $\mathrm{SR}(m, n)$ are of three types:

1. All adjacencies are $(j, k)$-adjacencies for fixed $j, k$. Now $|C| \leq n+1$.

2. $C=\left\{x+a e_{i} \mid i \in I\right\}$, where $a \in \mathbb{N}, 1 \leq a \leq n, x \in \mathbb{N}^{m}$ with $\sum x_{i}=n-a$, and $I \subseteq\{1, \ldots, m\}$. Now $|C| \leq m$.

3. $C=\left\{x-a e_{i} \mid i \in I\right\}$, where $a \in \mathbb{N}, a \geq 1, x \in \mathbb{N}^{m}$ with $\sum x_{i}=n+a$, $I \subseteq\{1, \ldots, m\}$, and $x_{i} \geq$ a for $i \in I$. Now $|C| \leq m$.

Proof Suppose $u, v, w$ are pairwise adjacent, not all $(j, k)$-adjacent for the same pair $(j, k)$. Then $u, v$ are $(i, j)$-adjacent, $u, w$ are $(i, k)$-adjacent, and $v, w$ are $(j, k)$ adjacent, for certain $i, j, k$. Now $u_{k}=v_{k}, u_{j}=w_{j}$ and $v_{i}=w_{i}$, so that $u=x+a e_{i}$, $v=x+a e_{j}, w=x+a e_{k}$, where $a>0$ or $a<0$.

Proposition 8.2 Let $m>1$ and $n>0$. Then the size of the largest clique in $\operatorname{SR}(m, n)$ is $\max (m, n+1)$. 
Proof For $n>0$, the $m$ vectors $n e_{i}$ are distinct and mutually adjacent, forming an $m$-clique. And for $m>1$, the $n+1$ vectors $a e_{1}+(n-a) e_{2}(0 \leq a \leq n)$ form an $(n+1)$-clique. Conversely, no larger cliques occur, as we just saw.

Fix a vertex $u$ of $\operatorname{SR}(m, n)$. We describe the structure of the local graph of $u$, that is the graph induced by $\operatorname{SR}(m, n)$ on the set $U$ of neighbors of $u$. If $v w$ is an edge in this local graph, then $u v w$ is a clique in $\operatorname{SR}(m, n)$, so we can invoke the above classification.

Lemma 8.3 (i) Any two adjacent vertices $u, v$ uniquely determine three cliques $C_{1}, C_{2}, C_{3}$ where $C_{i}$ is of type $i$ and $C_{i} \cap C_{j}=\{u, v\}$ for distinct $i, j \in\{1,2,3\}$, and $C_{1} \cup C_{2} \cup C_{3}$ contains all common neighbors of $u$ and $v$.

(ii) Fix a vertex $u$. For each $i \in\{1,2,3\}$, the cliques on u of type i form (after removal of $u$ ) a partition of the set $U$ of neighbors of $u$. Each edge in $U$ is contained in a unique such clique and hence has a unique type.

(iii) $\operatorname{SR}(m, n)$ does not contain an induced $K_{1,1,4}$.

Proof Let $C_{i}=C_{i}(u, v)(i=1,2,3)$ be the unique largest clique on $\{u, v\}$ of type $i$ $(i=1,2,3)$.

The set $U$ has a partition into $\left(\begin{array}{c}m \\ 2\end{array}\right)$ cliques of type 1 , where the $(j, k)$-clique has size $u_{j}+u_{k}$. (We check that $\sum_{j, k} u_{j}+u_{k}=(m-1) \sum_{j} u_{j}=(m-1) n$.)

The set $U$ has a partition into $n$ cliques of type 2, each of size $m-1$. Finally, $U$ has a partition into cliques of type 3. (If $v=u-a e_{j}+a e_{k}$ is a neighbor of $u$, then $C_{3}(u, v)=\left\{x-a e_{i} \mid i \in I\right\}$, where $x=u+a e_{k}$ and $I=\left\{i \mid 1 \leq i \leq m, x_{i} \geq a\right\}$.)

Lemma 8.4 Let $m, n \geq 3$, and fix a vertex $u$. Each neighbor $v$ of $u$ is contained in at most two maximal cliques precisely when u has only one nonzero coordinate.

Proof Suppose each point $v$ of $U$ is covered by at most two maximal cliques. Then one of the cliques of types 1 or 3 on $v$ in $U$ has size 1 . This means that whenever $u_{j}+u_{k} \geq 2$, we have $u_{i}=0$ for $i \neq j, k$. If $u_{j} \geq 2$, this means that $u$ has only one nonzero coordinate. If $u_{j}=u_{k}=1$, this means that $n=2$.

Suppose $m, n \geq 3$. We see that we can retrieve $V_{1}$ as the set of vertices that are locally the union of two cliques.

\section{Independence number}

It is known that $\mathrm{SR}(3, n)$ has independence number $\alpha(3, n)=\lfloor(2 n+3) / 3\rfloor$ (see $[11$, Problem 252], [1,10]). We determine $\alpha(m, 3)$.

\section{Proposition 9.1}

$$
\alpha(m, 3)= \begin{cases}\frac{1}{6}(m+1)(m+2) & \text { for } m \equiv \pm 1 \bmod 6 \\ \frac{1}{6} m(m+3) & \text { for } m \equiv 3 \bmod 6 \\ \frac{1}{6} m(m+2) & \text { for } m \equiv 0,4 \bmod 6 \\ \frac{1}{6}\left(m^{2}+2 m-2\right) & \text { for } m \equiv 2 \bmod 6 .\end{cases}
$$


Proof An easy upper bound is

$$
\alpha(m, 3) \leq m+\left\lfloor\frac{1}{3}\left(m\left\lfloor\frac{1}{2}(m-3)\right\rfloor+1\right)\right\rfloor .
$$

As follows: Count edges in $K_{m}$ covered by vertices of the independent set. No edge is covered twice because the corresponding vertices would be adjacent. A vertex $3 e_{i}$ (singleton) covers no edge. A vertex $2 e_{i}+e_{j}$ (pair) covers the edge $i j$. A vertex $e_{i}+e_{j}+e_{k}$ (triple) covers the edges $i j$ and $i k$ and $j k$. Nonadjacent vertices $3 e_{h}$ and $2 e_{i}+e_{j}$ have $h \neq i, j$. Nonadjacent vertices $2 e_{i}+e_{j}$ and $2 e_{k}+e_{l}$ have $i \neq k$ and $j \neq l$ and $\{i, j\} \neq\{k, l\}$. It follows that there are at most $m$ vertices of the forms $3 e_{h}$ and $2 e_{i}+e_{j}$. Since a triple takes 3 edges, and a pair only 1 , and a singleton 0 , we find an upper bound by assuming that there are 1 singleton and $m-1$ pairs ( 2 on each non-singleton). That leaves $m-3$ edges on each non-singleton, and 2 more on the singleton, for a maximum of $\frac{1}{2}(m-3)$ triples on each non-singleton, and 1 more on the singleton. Since each triple is counted thrice, there are at most $\frac{1}{3}\left(m\left\lfloor\frac{1}{2}(m-3)\right\rfloor+1\right)$ triples.

Separating the cases for $m(\bmod 6)$ yields precisely the values claimed, so they are upper bounds. But examples reaching the bounds can be constructed from Steiner triple systems.

If $m \equiv \pm 1(\bmod 6)$, then $\alpha(m, 3) \geq \frac{1}{6}(m+2)(m+1)$. [Take an $\operatorname{STS}(m+2)$, and delete two points $x, y$. The graph of noncovered edges has valency 2 , so is a union of cycles. Direct each cycle. Pick $2 e_{i}+e_{j}$ for each directed edge $(i, j)$. Let $x y z$ be a block. Pick $3 e_{z}$. This is a coclique of the indicated size that reaches the easy upper bound].

If $m \equiv 3(\bmod 6)$, then $\alpha(m, 3) \geq \frac{1}{6} m(m+3)$. (Take an STS $(m)$ with parallel class [e.g., a $\operatorname{KTS}(m)$ ] and view the triples in the parallel class as directed cycles).

If $m \equiv 0,2,4(\bmod 6)$, then the claimed value is obtained by shortening an example for $m+1$.

\section{Cospectral mates}

For $m \leq 2$ or $n \leq 2$, the graph $\operatorname{SR}(m, n)$ is complete or triangular and hence determined by its spectrum, except in the case of $m=7, n=2$ where it is isomorphic to the triangular graph $T(8)$, and cospectral with the three Chang graphs (cf. $[4,5])$. The graph $\mathrm{SR}(3,3)$ is 6-regular on 10 vertices, and we find that its complement is cubic with spectrum $3^{1} 2^{1} 1^{3}(-1)^{2}(-2)^{3}$. All integral cubic graphs are known, and $\operatorname{SR}(3,3)$ is uniquely determined by its spectrum, cf. [3], Sect. 3.8. We give some further cases where $\operatorname{SR}(m, n)$ is not determined by its spectrum.

Proposition 10.1 The graph $\mathrm{SR}(m, n)$ is not determined by its spectrum when $(a)$ $m=4$ and $n \geq 3$, or $(b) n=3$ and $m \geq 4$.

Proof Apply Godsil-McKay switching (cf. [3,7], 1.8.3, 14.2.3). Switch with respect to a 4-clique $B$ such that every vertex outside $B$ is adjacent to 0,2 or 4 vertices inside. If $m=4$, take $B=\{n 000,0 n 00,00 n 0,000 n\}$. If $n=3, m \geq 2$, take 
$B=\left\{a e_{1}+b e_{2} \mid a+b=3\right\}$. In both cases, every vertex outside $B$ is adjacent to 0 or 2 vertices inside. The switching operation preserves all edges and nonedges, except that it changes adjacency for pairs $b c$ with $b \in B, c \notin B$, and $c$ adjacent to 2 vertices of $B$, turning edges (resp. nonedges) into nonedges (resp. edges). The resulting graph has the same spectrum. We show that it is nonisomorphic to $\operatorname{SR}(m, n)$ for $m=4$, $n \geq 3$ and for $n=3, m \geq 4$.

In the former case, $B=V_{1}$. If switching does not change the isomorphism type, then $B$ must remain the $V_{1}$ of the new graph (since it is a single orbit of size $m$ contained in $V_{1} \cup V_{2}$ ). But after switching the common neighbors of $n 000$ and $0 n^{\prime} 10$ (with $n^{\prime}=n-1$ ) include the pairwise nonadjacent $0 n^{\prime} 01,01 n^{\prime} 0,001 n^{\prime}$, contradicting Lemma 8.4.

In the latter case, $B=\{3000 . ., 2100 . ., 1200 . ., 0300 .$.$\} . After switching, 3000..$ and 0300. are still in $V_{1}$ since their local graphs are $3 \times(m-1)$ grids. But $2100 .$. and 1200 .. are not, since the common neighbors of 2100 .. and 0030 .. include the pairwise nonadjacent $1200 . ., 1020 . ., 0210 \ldots$ And $1110 .$. is not, since the common neighbors of 1110.. and 0120.. include the pairwise nonadjacent 3000.., 1020.., 0111... So, there is no candidate for $V_{1}$.

There are at least 336 pairwise nonisomorphic graphs with spectrum $9^{1} 3^{4} 1^{3}(-1)^{6}$ $(-3)^{6}$, namely SR $(4,3)$ and the three graphs obtained by Godsil-McKay switching with respect to the 4-cliques $\{3000,0300,0030,0003\},\{0111,1011,1101,1110\}$ and $\{3000,2100,1200,0300\}$, and 332 further graphs obtained by repeated switching w.r.t. regular subgraphs of size 4 .

\section{The eigenspace of the smallest eigenvalue}

Fix $\pi \in \operatorname{Sym}(m)$, and let $a_{i}=\#\left\{j \mid i<j\right.$ and $\left.\pi_{i}>\pi_{j}\right\}$ for $1 \leq i \leq m$. Then $a=\left(a_{i}\right)$ is a vertex of $\operatorname{SR}(m, n)$ when $n$ is the number of inversions of $\pi$.

Say that $\sigma \in \operatorname{Sym}(m)$ is $\pi$-admissible if $a_{i}+i-\sigma_{i} \geq 0$ for $1 \leq i \leq m$. Let $\operatorname{Adm}(\pi)$ be the set of $\pi$-admissible permutations and define $x(\sigma)$ by $x(\sigma)_{i}=a_{i}+i-\sigma_{i}$. Then $\sigma \in \operatorname{Adm}(\pi)$ if and only if $x(\sigma)$ is a vertex of $\operatorname{SR}(m, n)$.

Theorem 11.1 (Martin and Wagner [9], Thm. 3.8) For each $\pi \in \operatorname{Sym}(m)$ with $n$ inversions, let

$$
F_{\pi}=\sum_{\sigma \in \operatorname{Adm}(\pi)} \operatorname{sgn}(\sigma) e_{x(\sigma)} .
$$

Then each $F_{\pi}$ is an eigenvector of $\operatorname{SR}(m, n)$ with eigenvalue $-n$, and the $F_{\pi}$ are linearly independent.

Theorem 11.2 (Martin and Wagner [9], Prop. 3.1) For $p, w \in \mathbb{R}^{m}$ such that $p+\sigma(w)$ for $\sigma \in \operatorname{Sym}(m)$ are pairwise distinct vertices of $\operatorname{SR}(m, n)$, define

$$
F_{p, w}=\sum_{\sigma \in \operatorname{Sym}(m)} \operatorname{sgn}(\sigma) e_{p+\sigma(w)} .
$$


Then each $F_{p, w}$ is an eigenvector of $\operatorname{SR}(m, n)$ with eigenvalue $-\left(\begin{array}{c}m \\ 2\end{array}\right)$, and for fixed $w$, the collection of all such $F_{p, w}$ is linearly independent.

Picking $w=\frac{1}{2}(1-m, 3-m, \ldots, m-3, m-1)$ yields the lower bound already mentioned earlier: The multiplicity of the eigenvalue $-\left(\begin{array}{c}m \\ 2\end{array}\right)$ is at least $\left(\begin{array}{c}n-\left(\begin{array}{c}m-1 \\ 2 \\ m-1\end{array}\right) \\ \text { a }\end{array}\right)$.

For the eigenvalue $-n$, it follows that its multiplicity is at least the number of elements in $\operatorname{Sym}(m)$ with precisely $n$ inversions, and one conjectures that equality holds.

The proof of Theorem 11.1 shows that for each $\pi \in \operatorname{Sym}(m)$ with $n$ inversions, the set $X_{\pi}=\{x(\sigma) \mid \sigma \in \operatorname{Adm}(\pi)\}$ induces a bipartite subgraph of $\operatorname{SR}(m, n)$ that is regular of valency $n$.

One may wonder what graphs $\Gamma(m, n, \pi)$ occur as induced subgraph on such a subset $X_{\pi}$ of the vertex set of $\operatorname{SR}(m, n)$. Given $\Gamma(m, n, \pi)$, one can find a $\pi^{\prime}$ such that $\Gamma\left(m+1, n, \pi^{\prime}\right) \simeq \Gamma(m, n, \pi)$, and a $\pi^{\prime \prime}$ such that $\Gamma\left(m+2, n+1, \pi^{\prime \prime}\right) \simeq$ $\Gamma(m, n, \pi) \times K_{2}$, where $\times$ denotes Cartesian product.

If $n=\left(\begin{array}{c}m \\ 2\end{array}\right)$, then there is a unique permutation $\pi_{0} \in \operatorname{Sym}(m)$ with $n$ inversions. It has $a=(m-1, m-2, \ldots, 0)$, and all $n$ ! permutations are $\pi_{0}$-admissible. The resulting graph $\Gamma\left(m,\left(\begin{array}{c}m \\ 2\end{array}\right), \pi_{0}\right)$ has as vertices all permutations of $(m-1, m-2, \ldots, 0)$, where two permutations are adjacent when they differ by a transposition. In other words, this is the Cayley graph $\operatorname{Cay}(\operatorname{Sym}(m), T)$, where $T$ is the set of transpositions in $\operatorname{Sym}(m)$.

Proposition 11.3 For any $\Gamma(m, n, \pi)$, where $m>2 n$, there is an isomorphic $\Gamma\left(2 n, n, \pi^{\prime}\right)$.

It follows that classifying all $\Gamma(m, n, \pi)$ for fixed $n$ is a finite job. Let $Q_{k}$ denote the $k$-cube. Using Sage, we find for $n=1$ that only $Q_{1}$ occurs, for $n=2$ that only $Q_{2}$ occurs, for $n=3$ that only $K_{3,3}$ and $Q_{3}$ occur, and for $n=4$ that only $K_{3,3} \times K_{2}$ and $Q_{4}$ occur. For larger $n$, one finds more complicated shapes.

It was conjectured in [9] that all graphs $\Gamma(m, n, \pi)$ have integral spectrum.

\section{Spectra for small $m$ or $n$}

If we fix a small value of $n$, we find a nice spectrum (eigenvalues and multiplicities are polynomials in $m, n$ ). If we fix a small value of $m \geq 3$, we get a messy result (also congruence conditions play a rôle). Below, multiplicities are written as exponents.

For $n=0$, the spectrum is $0^{1}$.

For $n=1$, the spectrum is $(m-1)^{1},(-1)^{m-1}$.

For $n=2$, the spectrum is $(2 m-2)^{1},(m-3)^{m},(-2)^{\text {rest }}$.

For $n=3$, the spectrum is $(3 m-3)^{1},(2 m-5)^{m},(m-3)^{m-1},(m-5)^{\left(\begin{array}{c}m \\ 2\end{array}\right)},(-3)^{\text {rest }}$.

(See below.)

For $n=4$, the spectrum is $(4 m-4)^{1},(3 m-7)^{m},(2 m-5)^{m},(2 m-8)\left(\begin{array}{c}m \\ 2\end{array}\right)$, $(m-4)\left(\begin{array}{c}m \\ 2\end{array}\right)-1,(m-6)\left(\begin{array}{c}m \\ 2\end{array}\right),(m-7)\left(\begin{array}{c}m \\ 3\end{array}\right),(-4)^{\text {rest }}$. (See below.)

For $n=5$, the spectrum may be $(5 m-5)^{1},(4 m-9)^{m},(3 m-7)^{m}$, $(3 m-11)^{\left(\begin{array}{c}m \\ 2\end{array}\right)},(2 m-5)^{m-1},(2 m-7)^{\left(\begin{array}{c}m \\ 2\end{array}\right)},(2 m-9)^{\left(\begin{array}{c}m \\ 2\end{array}\right)},(2 m-11)^{\left(\begin{array}{c}m \\ 3\end{array}\right)},(m-5)^{\left(\begin{array}{c}m \\ 3\end{array}\right)-1}$, $(m-6)^{m(m-2)},(m-8)^{2\left(\begin{array}{c}m \\ 3\end{array}\right)},(m-9)^{\left(\begin{array}{c}m \\ 4\end{array}\right)},(-5)^{\text {rest }}$. 
For $m=1$, the spectrum is $0^{1}$.

For $m=2$, the spectrum is $n^{1},(-1)^{n}$.

For $m=3$, the spectrum is $(2 n)^{1}, b^{3}$ (for all $b \in \mathbb{Z}$ with $-2 \leq b \leq n-2$ ),

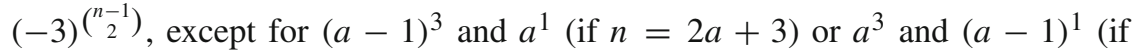
$n=2 a+4)$ (Martin and Wagner [9]).

For $m=4$, we give some values in Table 1 .

Let $a^{m} \downarrow b$ denote sequence of eigenvalues and multiplicities found as follows: The eigenvalues are the integers $c$ with $a \geq c \geq b$, where the first multiplicity is $m$, and each following multiplicity is 2 larger for even $c$, and 10 larger for odd $c$. Now the conjectured spectrum of $\operatorname{SR}(4, n), n \geq 6, n \neq 7$ consists of

(i) $(3 n)^{1}$,

(ii) $b^{4}$ for all odd integers $b$, where $2 n-3 \geq b \geq n-1$, (iii)

\begin{tabular}{ll}
$n=2 r$ & $(n-4)^{3 n-1},(n-6)^{6},(n-7)^{16} \downarrow(n-8) / 2$ \\
$n=2 r+1$ & $(n-2)^{3},(n-4)^{3 n-3},(n-6)^{9},(n-7)^{12} \downarrow(n-7) / 2$ \\
\hline
\end{tabular}

(iv) for $q=\lceil n / 3-4\rceil$ :

\begin{tabular}{ll}
\hline$n=4 s$ & $(2 s-5)^{3 n-12},(2 s-6)^{3 n-26} \downarrow q$ \\
$n=4 s+1$ & $(2 s-4)^{3 n-7},(2 s-5)^{3 n-21},(2 s-6)^{3 n-23} \downarrow q$ \\
$n=4 s+2$ & $(2 s-4)^{3 n-16},(2 s-5)^{3 n-22 \downarrow q}$ \\
$n=4 s+3$ & $(2 s-3)^{3 n-3},(2 s-4)^{3 n-25},(2 s-5)^{3 n-19} \downarrow q$ \\
\hline
\end{tabular}

(v) if $n \equiv 0(\bmod 3)$ one additional eigenvalue $n / 3-4$, (vi)

\begin{tabular}{ll}
\hline$n=6 t$ & $(2 t-5)^{4 n-12},(2 t-6)^{4 n-16},(2 t-7)^{4 n-16} \downarrow(-5)$ \\
$n=6 t+1$ & $(2 t-4)^{4 n-32},(2 t-5)^{4 n-7},(2 t-6)^{4 n-20},(2 t-7)^{4 n-14} \downarrow(-5)$ \\
$n=6 t+2$ & $(2 t-4)^{4 n-24},(2 t-5)^{4 n-8},(2 t-6)^{4 n-21},(2 t-7)^{4 n-12} \downarrow(-5)$ \\
$n=6 t+3$ & $(2 t-4)^{4 n-16},(2 t-5)^{4 n-12},(2 t-6)^{4 n-20} \downarrow(-5)$ \\
$n=6 t+4$ & $(2 t-3)^{4 n-28},(2 t-4)^{4 n-11},(2 t-5)^{4 n-16},(2 t-6)^{4 n-18} \downarrow(-5)$ \\
$n=6 t+5$ & $(2 t-3)^{4 n-20},(2 t-4)^{4 n-12},(2 t-5)^{4 n-17},(2 t-6)^{4 n-16} \downarrow(-5)$ \\
\hline
\end{tabular}

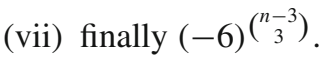

For example, -5 has multiplicity $6 n-28$.

The above is trivial for $m<3$ or $n<3$. It was done in [9] for $m=3$ and will be done below for $n=3,4$. The suggested spectra for $n=5$ were extrapolated from small cases. We have not attempted to write down a proof. 


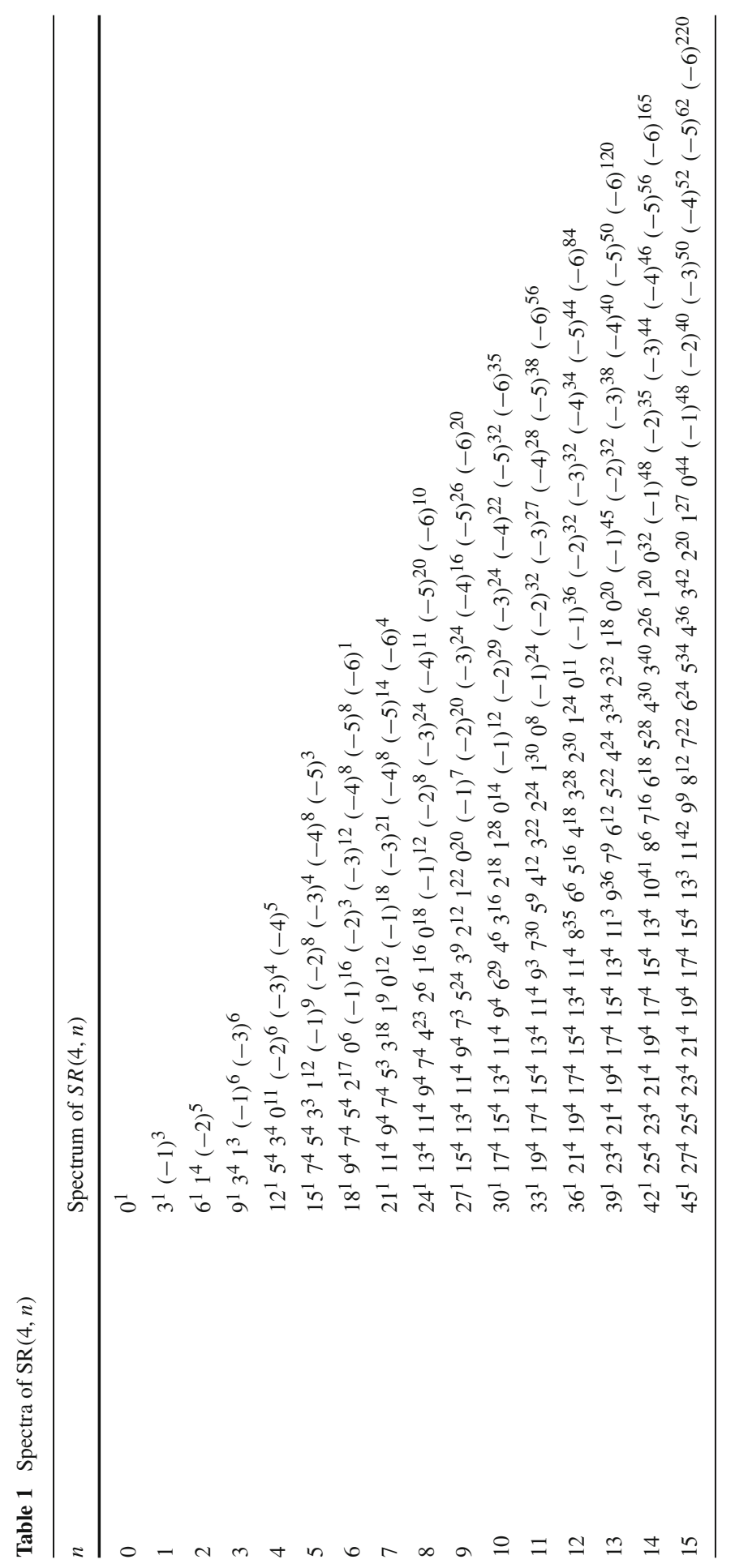


Proposition 12.1 The graph $\mathrm{SR}(m, 3)$ has spectrum $(3 m-3)^{1},(2 m-5)^{m},(m-$ $3)^{m-1},(m-5)^{\left(\begin{array}{c}m \\ 2\end{array}\right)},(-3)^{m\left(m^{2}-7\right) / 6}$.

Proof In view of the common part of the spectra of $\mathrm{SR}(m, 3)$ and $J(m+2,3)$, and the fact that $m\left(m^{2}-7\right) / 6$ is the coefficient of $t^{3}$ in $\prod_{i=2}^{m}\left(1+t+\cdots+t^{i-1}\right)$ (for $m \geq 3$ ), and the fact that the stated multiplicities sum to the total number of vertices, it follows that we only have to show the presence of the part $(m-3)^{m-1}$.

Fix an index $h, 1 \leq h \leq m$ and consider the vector $p$ indexed by the vertices that is 1 in vertices $2 e_{h}+e_{i}$ and -1 on vertices $e_{h}+2 e_{i}$ and 0 elsewhere. One checks that this is an eigenvector with eigenvalue $m-3$, and the $m$ vectors defined in this way have only a single dependency (namely, they sum to 0 ).

Proposition 12.2 The graph SR $(m, 4)$ has spectrum $(4 m-4)^{1},(3 m-7)^{m},(2 m-$ $5)^{m},(2 m-8)^{\left(\begin{array}{c}m \\ 2\end{array}\right)},(m-4)^{\left(\begin{array}{c}m \\ 2\end{array}\right)-1},(m-6)^{\left(\begin{array}{c}m \\ 2\end{array}\right)},(m-7)^{\left(\begin{array}{c}m \\ 3\end{array}\right)},(-4)^{r}$ where $r=$ $m\left(m^{3}+2 m^{2}-13 m-14\right) / 24$.

Proof In view of the common part of the spectra of SR $(m, 4)$ and $J(m+3,4)$, and the fact that $r$ is the coefficient of $t^{4}$ in $\prod_{i=2}^{m}\left(1+t+\cdots+t^{i-1}\right.$ ) (for $m \geq 4$ ), and the fact that the stated multiplicities sum to the total number of vertices, it follows that we only have to show the presence of the part $(2 m-5)^{m},(m-4)^{\left(\begin{array}{c}m \\ 2\end{array}\right)-1},(m-6){ }^{\left(\begin{array}{c}m \\ 2\end{array}\right)}$ of the spectrum.

Any eigenvector for one of these eigenvalues sums to zero on each part of the fine equitable partition found earlier, that is, on each set of vertices with given support. Since there are unique vertices with support of sizes 1 or 4 , these eigenvectors are 0 there, and we need only look at the vertices $3 e_{i}+e_{j}$ and $2 e_{i}+2 e_{j}$ and $2 e_{i}+e_{j}+e_{k}$.

Fix an index $h, 1 \leq h \leq m$ and consider the vector $p$ (indexed by the vertices) that vanishes on each vertex where $h$ is not in the support, is -1 on $2 e_{h}+2 e_{i}$ and on $3 e_{h}+e_{i}$, is 2 on $e_{h}+3 e_{i}$, is -2 on $2 e_{h}+e_{i}+e_{j}$, and is 1 on $e_{h}+2 e_{i}+e_{j}$. One checks that this is an eigenvector with eigenvalue $2 m-5$ and that the $m$ vectors defined in this way are linearly independent. That settles the part $(2 m-5)^{m}$.

Fix a pair of indices $h, i, 1 \leq h<i \leq m$, and consider the vector $p$ (indexed by the vertices) that is 1 on $e_{h}+3 e_{j}, 2 e_{i}+2 e_{j}$ and $2 e_{h}+e_{i}+e_{j}$, is -1 on $e_{i}+3 e_{j}, 2 e_{h}+2 e_{j}$ and $e_{h}+2 e_{i}+e_{j}$, and is 0 elsewhere. One checks that this is an eigenvector with eigenvalue $m-6$ and that the $\left(\begin{array}{c}m \\ 2\end{array}\right)$ vectors defined in this way are linearly independent. That settles the part $(m-6)\left(\begin{array}{c}m \\ 2\end{array}\right)$.

Having found all desired eigenvalues except one, it is not necessary to construct eigenvectors for the final one, since checking $\sum \theta=\operatorname{tr} A=0$ and $\sum \theta^{2}=\operatorname{tr} A^{2}=v k$ suffices.

Open Access This article is distributed under the terms of the Creative Commons Attribution 4.0 International License (http://creativecommons.org/licenses/by/4.0/), which permits unrestricted use, distribution, and reproduction in any medium, provided you give appropriate credit to the original author(s) and the source, provide a link to the Creative Commons license, and indicate if changes were made. 


\section{References}

1. Blackburn, S.R., Paterson, M.B., Stinson, D.R.: Putting dots in triangles. J. Comb. Math. Combin. Comput. 78, 23-32 (2011)

2. Brouwer, A.E., Cohen, A.M., Neumaier, A.: Distance-Regular Graphs. Springer, New York (1989)

3. Brouwer, A.E., Haemers, W.H.: Spectra of Graphs. Springer, New York (2012)

4. Chang, L.C.: The uniqueness and nonuniqueness of the triangular association scheme. Sci. Rec. 3, 604-613 (1959)

5. Chang, L.C.: Association schemes of partially balanced block designs with parameters $v=28, n_{1}=$ $12, n_{2}=15$ and $p_{11}^{2}=4$. Sci. Rec. 4, 12-18 (1960)

6. Elkies, N.D.: Mathoverflow (2012). http://mathoverflow.net/questions/103540/hexagonal-rooks

7. Godsil, C.D., McKay, B.D.: Constructing cospectral graphs. Aequ. Math. 25, 257-268 (1982)

8. Humphreys, J.E.: Reflection Groups and Coxeter Groups. Cambridge University Press, Cambridge (1990)

9. Martin, J.L., Wagner, J.D.: On the spectra of simplicial rook graphs, arXiv:1209.3493 / Graphs and Combinatorics, on-line 18 May 2014/pp. 373-386 In: Proceedings of the 25th International Conference on Formal Power Series and Algebraic Combinatorics (FPSAC 2013), Discrete Math. Theor. Comput. Sci. Proc, Nancy (2013)

10. Nivasch, G., Lev, E.: Non-attacking queens on a triangle. Math. Mag. 78, 399-403 (2005)

11. Vaderlind, P., Guy, R.K., Larson, L.C.: The Inquisitive Problem Solver. MAA Problem Book Series. Mathematical Association of America, Washington, DC (2002) 